\title{
BLOCKCHAIN APPLICATIONS AND SUSTAINABILITY ISSUES
}

\author{
Roberto Leonardo Rana ${ }^{1}$, Pasquale Giungato ${ }^{2 *}$, Angela Tarabella ${ }^{3}$ \\ and Caterina Tricase ${ }^{4}$ \\ 1), 4) University of Foggia, Italy; ${ }^{2)}$ University of Bari, Italy \\ ${ }^{3)}$ University of Pisa, Italy
}

Please cite this article as:

Article History

Rana, R.L., Giungato, P., Tarabella, A. and Tricase, C.,

Received: 9 August 2019

2019. Blockchain Applications and Sustainability Issues.

Amfiteatru Economic, 21(Special Issue No. 13),

Revised: 24 September 2019

pp. 861-870.

DOI: $10.24818 / \mathrm{EA} / 2019 / \mathrm{S} 13 / 861$

\begin{abstract}
Blockchain, a distributed and democratically-sustained public register of the transactions of the digital currency "Bitcoin", proposed by Satoshi Nakamoto a pseudonym of a hided developer in 2009, has been the driver of a huge number of initiatives devoted to develop and implement a peer-to-peer distributed database, and with no central authority created as an open source software. Expansion of Bitcoin, as well as other digital currencies, has been due to lower transaction costs, high security protocols and lack of inflation with respect to fiat money and no need of a clearing entity or a central bank. Although, environmental issues related to the use of this currency and, in particular, in the energy consumes, have been raised by the scientific community, but no signals of limiting factors have been detected until now. The hidden in the wings blockchain technology has been recognized the driver of innovation in various fields, contributing to create a more sustainable world. The purpose of this paper is to describe both the recent trends in the applications of the blockchain technology in the cryptocurrencies market and the new projects considering the environmental sustainability (energy consumption, materials depletion) and social impacts. The contribute of blockchain in reducing and accelerating bureaucracy and incentivizing environmentally friendly behaviour, has been discussed. Although the environmental issues related to the energy consumed in Bitcoin mining may scare companies in adopting the new technology, there is no evidence of limiting factors or carrying capacity of the entire system. In future the development of a less energy intensive alternative for validating the blocks to stack in the distributed database, would render the blockchain the ideal candidate for the applicability of sustainability paradigms in the economic, environmental and social sectors.
\end{abstract}

Keywords: blockchain, bitcoin, sustainability, energy consumption, cryptocurrency.

JEL Classification: O33.

* Corresponding author, Pasquale Giungato - pasquale.giungato@uniba.it 


\section{Introduction}

The first digital money created by an hided developer, whose name was Satoshi Nakamoto opened the door to a new digital revolution, introducing not only a cryptocurrency, as it was later called, but disclosed to the public a new democratically-sustained ledger of all transactions that would have had interesting practical applications also in other fields. The innovation introduced by Nakamoto (but it seems there should be a group hided under this pseudonym) represents a currency that bypasses the official way to produce and exchange money, based on a central bank or a clearing authority, and uses a peer-to-peer payment system realised as an open source software. This virtual money run thanks to the blockchain technology that create a distributed public register containing all Bitcoin transactions. As a result, all peer-to-peer money transactions are registered and stored without any changing in a secure way with no need of a central bank, with a mechanism of consensus among all the network nodes which share a portfolio of Bitcoins. Following the success of Bitcoins, a huge number of new cryptocurrencies have been introduced (almost 2,000) since 2009 and more than 600 of which are actively traded today. Most of them are duplicates of Bitcoin, while others derive from significant innovations of the blockchain technology. Cryptocurrencies are principally used as ways of exchange payments and for speculation. Other common uses include payment rail for non-expensive cross borders money transfer and various non-monetary uses such as timestamp (El Bahrawy et al., 2017). Although, these cryptocurrencies have produced a market capitalization equal to $\$ 253.453 .163 .612$ (Sep 04, 2019) some issues remain unsolved as long-term sustainability and blockchain latency improvements (Michel and Hudon, 2015). Despite of numerous issues related to the cryptocurrency market, the related technology of blockchain could represent a significant tool contributing to create a more sustainable world. The aim of this paper is, in the first paragraph to illustrate the operating mechanism of the virtual money, to understand better blockchain strengths and weaknesses; in the second part to evaluate the Bitcoin and blockchain technology sustainability, considering the environmental and social impacts due to energy consumption and market diffusion compared to fiat currency, the third part on discussion of the results.

\section{Bitcoin and blockchain technology}

Bitcoin is a cryptocurrency based on the blockchain technology, which make use of secure cryptographic algorithms supported by a peer-to-peer network. Blockchain is based on the following assumption: it rely on a peer-to-peer network and there is no need of a central server, it has a distributed consensus protocol that consists of digital signature and cryptography based on asymmetric public key mechanism, a timestamp system to make transaction traceable in a peer-to-peer network in which all nodes in the network are equal peers. To maintain and sustain the network the technology makes use of a remuneration in terms of a reward in Bitcoins given to the node of the network which resolve a simple but computing intensive task, necessary to validate a block of transactions (whose validity has been verified by the software), append it to the blockchain to disseminate in the network of all connected computers. As a limited and scarce resource the rate of emission of new Bitcoins or "Bitcoin mining", resembles the iron or gold ores mining, because it has been modulated by an algorithmic limitation starting from 50 unities with an increase rate slowing down constantly to resemble the cumulated production of a natural resource: the emission rate will halve after the issue of 10.5 million Bitcoins, and will halve again after 
the emission of $15,750,000$ Bitcoins, reaching a limited capped value (carrying capacity) of 21 million Bitcoins, the maximum reachable value of the cumulated Bitcoins production (Karger, 1997). Nowadays there are 17,944,800 (Sept. 19, 2019) Bitcoins mined. The number depends on the mechanism of the algorithm which follows a geometric series (1).

$N=\sum_{n} \frac{210,000 \times 50}{2^{\mathrm{n}}}$

In equation (1), $\mathrm{N}=$ cumulated number of Bitcoins at time $\mathrm{n}$ and the limit for $\mathrm{n}$ approaching $+\infty$ is equal to 21 million. The cumulated Bitcoin mining, especially in more recent years follow a statistical distribution like the geometric one (see also "Bitcoin in circulation" on: www.blockchain.info).

Blockchain technology holds transactions grouped in blocks and shared and validated by all nodes through a distributed consensus protocol, the Proof of Work (POW) consensus (Blockchain.info, 2019). Each node in the blockchain stores a complete or partial copy of the database containing the blocks with the entire bitcoin transaction history. New validated transactions are attached to the chain and propagates in the network from a source (input) to a destination (output) which are not connected to portfolios nor are balanced by a central server. Each node of the blockchain network verifies the block of transactions it composed, propagates only valid transactions, with the consensus protocol building a valid transactions pool. The node firstly checks the syntax and structure of the transaction, verify its validity, by computing the POW or a cryptographic hash of the block meeting certain constraints. To comply to security issues this verification checksum for the block, is a one-way type algorithm. It is easy to compute a hash of a given block, but difficult to build a block that matches a given hash, as this task require a computational power far beyond the possibility of a single node. The power of the idea consists in the collision resistance of the hashing algorithm as it is very difficult to find two blocks that produce the same hash. Finding a valid hash which connects the elastic constraints imposed by the blockchain, is a computeintensive task executed but it consists of a brute-force simply task (Rana et al., 2019). The nodes of the blockchain network really compete in finding a valid hash that can be validated and stacked in the blockchain and the first one finding a valid hash, is rewarded with a percentage of new mined Bitcoins and the nodes are really motivated to sustain the blockchain network. Once a valid block is stacked in the blockchain a receiving node verify the validity of the computed hash and if successful, the mining process is stopped for all managing the same transactions and the competition will start with mining for a new block. In the eventuality that multiple nodes will simultaneously generate a valid block, the appeared fork will be resolved in a democratically manner as one branch will not receive the consensus of the other nodes in stacking the block (Vranken, 2018). The blockchain rely heavily on the consensus of the network as it is essential to achieve the unanimous confirmation of the verified transactions by the nodes involved in the blockchain network. The Proof-of-work is a brute force computational task in which the node to verify the validity of the block of transaction and stack the block to the blockchain, must solve a computationally difficult but easily verified SHA256 task using its own computational power, or the computational power of a network of parallel computing CPUs. The task consists in finding a random number called "Nonce" which is added information about the transactions or the blocks of transactions to be grouped by the SHA256 hash algorithm

Vol. $21 \cdot$ Special Issue No. $13 \cdot$ November 2019 
twice in succession, to be sure of the irreversibility of the operation. The POW consensus mechanisms will allow a remuneration in Bitcoins, or part of it, at the expenses of computational power. Theoretically a malicious node should take almost the $51 \%$ of the computational power of the entire network of blockchain to get successful in an attempt to generate a fake block and realize a double spending attack. Double-spending issues are well known studied as also the presence of malicious nodes in the Generals Byzantine fault issues (Fan et al., 2013; Fedotova and Veltri, 2006; Reischuk, 1985; Lamport, 1983). These two computer technology conditions appeared in a computer network when some of the nodes of the network are malicious ones as inserts unreliable information: to be sure that the entire will agree on a concerted strategy to avoid a collapse, the system should be reinforced. The presence of malicious nodes recalls that of the Byzantine generals who reported fakes news on war strategies to their colleagues, to invalidate the final victory. The bitcoin mining has fostered nodes to increase dramatically its computational power that now has surpassed some world's supercomputers, or BOINC networks but mining rate is really constant as its emission rate is regulated by a mechanism of adjusting difficulty in searching of a valid nonce values with the aforementioned brute-force approach; the adjusting mechanisms in searching the right hash for the consensus operates every 2016 blocks, ensuring an average interval of 10 minutes between linked blocks. Research is directed to stop the rush to increase the computational power, to be successful in validating a new block to be rewarded, by introducing new but reliable consensus protocols.

\section{Bitcoins and blockchain sustainability issues}

According to Giungato et al. (2017) although cryptocurrencies seems to be environmentally unsustainable, since it consumes high amount of energy to mine and to perform a transaction, there is no evidence of a carrying capacity of the system. The trend of the bitcoin mining does not resemble that of a limited resource like fossil fuels or copper, gold (for instance a logistic equation like that used in crude oil consumption and production forecasts made by geologists) and the trend of bitcoin mining is exactly that forecasted by equation (1) with variation in slope predicted by the adjusting algorithm. Despite this lack of evidence of a limiting factor in bitcoin mining the International Energy Agency (2017) estimates that electricity used for mining Bitcoin it is approximately less than $1 / 40^{\text {th }}$ of $1 \%$ of global electricity consumption in 2016. This massive fossil fuels consumption implicates a default on the Paris Agreement climate change commitments and contribute to increase the climate change processes. Recently, de Vries (2018) has proposed an indicator using and empirical methodology still not validate by scholars, called Bitcoin Energy Consumption Index that estimate and predict energy consumed to mine this virtual money. The author assessed in the 2019 (until September) an average energy consumption equal to 73.21TWh that correspond to the release of $34.73 \mathrm{MtCO}_{2}$ or $\mathrm{CO}_{2}$ emitted by Denmark in the same year (de Vries, 2019a).

In figure no. 1 is shown two trends of $\mathrm{CO}_{2}$ emitted by energy consumed for Bitcoin transactions. According to de Vries (2019b) the energy consumption is an estimation, so the blue and yellow line consider the maximum and minus quantity of $\mathrm{CO}_{2}$ release by all transactions. In both trends there is an increased from 2017 to the end of 2018 followed by a decrease. In the begin of 2019 a new increase is observed. 


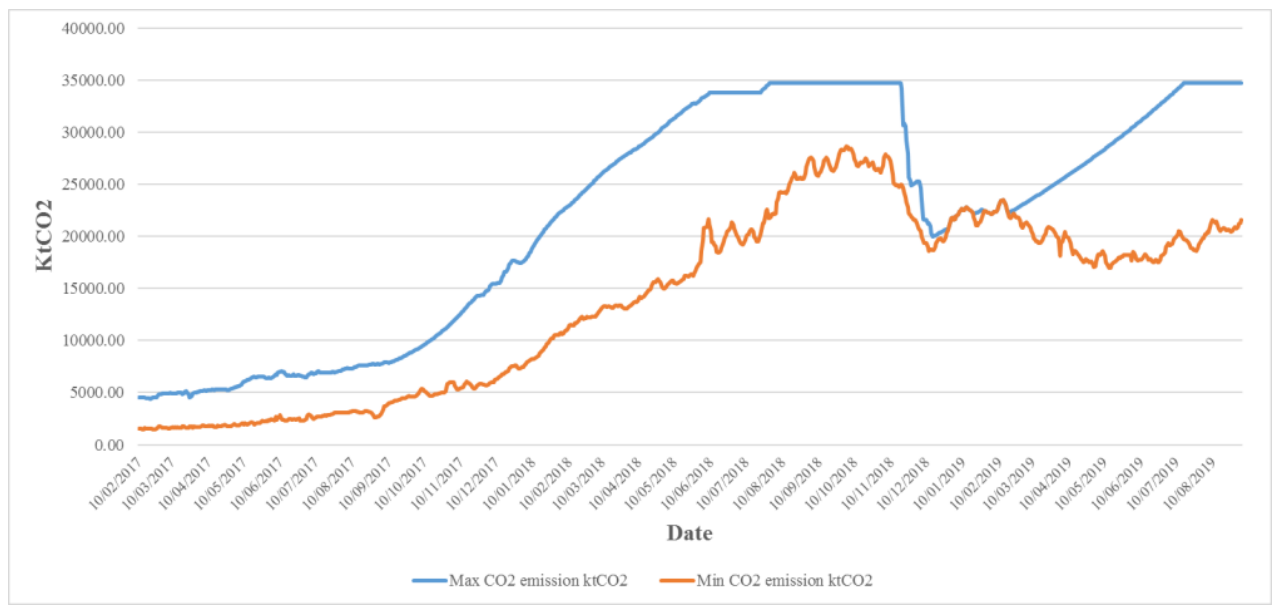

Figure no. 1. Trend of carbon footprint of Bitcoin

\section{Source: Adapted after de Vries, 2019a}

De Vries (2019b) underline that Bitcoin network, and in general cryptocurrencies, generate significant quantities of electronic waste (e-waste). The author state that the reason for this problem is due to Bitcoin mining which required specialized hardware and becomes obsolete about every year and a half. So, less efficient devices used to mine cryptocurrency will be pressured out of the market sooner or later becuse they cannot compete with more efficient hardwares. De Vries has calculated that in the last 9 months the amount of Bitcoin electronic waste produced is about $10 \mathrm{Mt}$ a quantity produced by Luxemburg in the same period of time. Moreover, comparing the electronic waste generated by one Bitcoin transaction with one VISA transaction the former produce about $80 \mathrm{~g}$ while the latter 20,000 time less (more than $0.4 \mathrm{mg}$ ). Because the Bitcoin mining activities are going to increase in the coming years the only way to make this money more sustainable is to substitute its mining mechanism with new one or using an array of alternative cryptocurrencies.

Another interesting environmental aspect in which the blockchain can offer a valid alternative to the actual one, is represented by the carbon off-set trading, in particular, for climate change mitigation. 2015 Paris Climate Agreement will depend on functioning of the carbon and energy markets, in the aim to use carbon tax as the leverage to mitigate fossils consumption. This approach is called "neoclassical" and the equilibrium is reached when taxes balance the avoided damages. Obviously for the success of the global policy this system should be implemented and accepted worldwide, involving a global consensus among parties that only an accepted technology as the blockchain can assure. This market is quite different from a cryptocurrency as is characterized exclusively by trading services, and the hypothetic cryptocurrency is replaced by a carbon offset/credit. The definition of this currency may be the service of preventing one metric tonne of carbon dioxide equivalent $\left(\mathrm{CO}_{2}\right.$-eq) from entering the atmosphere. Carbon stock-taking for low-carbon projects are more vulnerable to frauds as they receive carbon offsets/credits as revenue for sequestering carbon or for reducing carbon emissions, is managed at a local scale and may be sensible to manipulations (Chen, 2018). 


\section{Results and discussions}

The bitcoin system was born after the global financial crisis with massive interventions by financial and non-financial institutions, in order to remove the risk of default of the entire global banking system. In this context, bitcoin appears as an innovative tool to eliminate the intermediation of banks and financial institutions and create a decentralized system. About its innovativeness many believe that it is limited and that the benefits are rather limited compared to the present electronic currencies. Indeed, bitcoin makes it possible to add up the advantages of cash and electronic payment systems (Alshamsi and Andras, 2019). In particular it allows, in summary, to:

- carry out transactions guaranteed by a certain level of anonymity;

- avoid the use of traditional financial institutions for the production and management of the currency;

- make payments at a distance;

- reduce transaction costs.

But despite these undeniable benefits, Bitcoin has not yet imposed itself on traditional payment systems. It presents a strong acceptance limit, in the sense that it can be spent for the purchase of goods and services online only in special sites that provide payments with virtual currency; its use is tied to the availability of a technology necessary for access not at the moment universally widespread. This limitation is a huge disadvantage both for the differences between countries and for the difficulty of access to the most modern technologies by the poorest section of the population. Risks related to transactions regulated by single individuals and difficulties to monitor as anonymous and with poor guarantee and protection in the event of disputes, risks of possible hacking of the sites that deal with exchange services, should also be considered.

Bitcoins are innovative but poorly understood systems, they owe their existence almost exclusively to the trust that users place in them and in the possibility of receiving huge economic returns for small investments. The Bitcoin system has thus become a highly speculative financial instrument with the possibility that cycles and speculative bubbles can be generated as in traditional financial markets.

In fact, bitcoin prices seem to depend more on media attention than on other factors.

In the choice of this cryptocurrency the socio-cultural aspects that some studies have shown have a determining role. In particular Abraham et al., 2019 analyzed the factors that influence the degree of penetration and acceptance of bitcoin at various individual and collective levels mainly related to the perception and acceptance of risk, the degree of safety and ease of use. Certainly it cannot be omitted that bitcoins generate negative externalities from an environmental point of view due to the inefficient use of energy sources used for the validation of transactions and are often used for non-transparent operations, criminal and illicit aims. Cryptocurrencies and, in particular Bitcoins, are considered close to the substantive such as anonymity and illegal although is not true in principle since all virtual transactions are public and permanent on the web. In fact, transparency and traceability is its characteristic, where all money transaction procedure take place on the web and not depend on a centralized organization. In addition, all transactions process required a distributed database that is a public peer-to-peer archive where all the virtual banking operations are 
shared among all the Bitcoin's holders. The security system is assured by a specific procedure called "digital distributed consensus" or Blockchain where all information can never be removed and stored in a specific archive. Consequently, this technology consists in a type of share and digital economy where all banking operations are traceable among different economic entities. Rana et al. (2019) state that this technology may open new and challenging opportunities to the economic system since it could overcome the problem connected to the huge bill that companies pay for maintaining paper-based archives of transactions. The flexibility of this technology apart from contribute to promote several projects in all economic sectors and to improve banking and financial systems it could has implications on the social aspects for many public and private organizations. (D. Tapscott and A. Tapscott, 2017). For instance, this technology has been suggested in health sector to share among doctors and specialists, data of their patients. In this way, records can be tracked and stored endless properly in a digital databank (i.e. cloud structure). Moreover, this information can be useful to improve research activities since can facilitate exchange of data between research groups and health care institutions. The security and privacy are protected inasmuch the blockchain technology offers a cryptographic protocol, which identify and authenticate users who access to medical information, keeping a trace of all their activities conduct (Xia et al., 2017).

According to Mikroyannidis et al. (2018) this technology is revolutionising also the lifelong learning in many ways. Indeed, blockchain could contribute to reorganize educational content and, in particular the training services online as follow: 1) to register and pay for these services; 2) to supervise tutoring activities; 3) to get certification for what learned; 4) to know how this certification influence the career of a worker. Moreover, the authors affirm that this technology offers to an educational institution full control and responsibility over their learning processes.

Electricity production and distribution is another sector where the blockchain technology could improve its management. The last decades the increase diffusion of new technology such as micro-wind, photovoltaic panels, micro hydropower generation system is quickly changing supply electricity from centralized grid to spread micro-grid at citizen's level (smart grid). Therefore, citizens are transforming from consumers to consumer-producer which reducing their electricity bills selling their excess power to other local users. In this contest the blockchain could improve the energy supply and management of smart grid among local users and producers (Green and Newman, 2017). The consumer-producers needs a technology for managing energy trading that ensure data exchanges characterised by a decentralized server with a public record book. In this regard, some enterprises or foundation such as Grid Singularity and Solar Coin are utilizing the blockchain-based energy trading system to better satisfy the practical requirements of modern power systems among citizen's utilities and microgrids (Wang et al., 2019). Although, this technology still has some problems to be solved (for example, the lack of regulatory system) it can help consumer-producers to managing energy produced or used by using a decentralised energy data exchange platform (Rutkin, 2016). For example, Giungato et al. (2019) illustrate an innovative application blockchain who consists in DNS (domain name system) project called "Blockstack". This decentralised technology DNS is a repository of web addressInternet Protocol address. Blockchain technology represents a different mapping DNS-like system, which, replace DNS root servers in resettling domain names into IP addresses. The goal of developers is to substitute servers controlled by corporations and governments with Blockchain DNS introducing decentralization, decreasing censorship resistance and increasing security and privacy events (Ali, 2016). Another alternative application of 
blockchain is in TLS (transport layer security) technology a cryptographic protocol that assure communications security (web browsing, web-fax, email, voice-over-IP (VoIP) or instant messaging) over nodes of a computer network. In this case, the use of security blockchain consensus can improve and decentralize TLS certificate validation.

Moreover, blockchain technology can improve E-commerce and governance within a city (Subramanian, 2018) or a smart city (Sun at al., 2016) so that it is possible democratize sharing services and trust is not carried out by a person, but it is spread on the entire population and the central authorities is substitute by a peer-to-peer network. For instance, in such a democratized framework, governments cannot influence an election and companies cannot unilaterally break or change the rules of the system. In particular, Sun et al. (2016) state that blockchain can contribute to develop Internet of Things (IoT) devices characterised by trust-free transactions, and contracts can be captured using a specific code which automatically carry out the obligations that parties have committed to in an agreement.

\section{Conclusions}

With the pseudonym "Satoshi Nakamoto" a hided developer, or a team of them, launched on the internet a digital currency named Bitcoin based on a peer-to-peer distributed payment system managed by an open source software. To sustain the network a miningwith-rewarding system, ensured transferring of this virtual money by cryptographic connections among virtual wallets, for this reason the Bitcoin was also called "cryptocurrency". The underlying technology, the "blockchain", has received increasing attention among the scientific community, and later by industrial companies, due to some crucial characteristics: decentralization (as it is a peer-to-peer network and there is no need of a central server), distributed consensus protocol, digital signature and secure cryptographic based mechanism, peer-to-peer transaction based on decentralized network in a distributed systems. Although these unique characteristics have determined the spreading of cryptocurrencies as virtual moneys, some concerns raised about their environmental sustainability since they require computational power and therefore, not negligible amounts of energy to sustain and maintain the payment system. Mining of Bitcoins and sustaining of blockchain in general, depend upon computational power made available by the nodes of the network and used to solve the proof-of-work rebus to validate a block of transaction and stack it to the democratically sustained database; to date there exist no proof of the existence of limiting factors in energy requirements but replacement of all the actual monetary mass into cryptocurrencies could be impossible and cryptocurrencies will occupy niche parts of the monetary market. Blockchain has receiving increasing attention in the scientific community and technological firms in sharing medical data, in managing energy micro grids among producers and consumers at a citizen's level, in contract management among companies, for file signatures, voting procedures, notary services, and application are growing day to day.

\section{References}

Abraham, J., Sutiksno, D.U., Kurniasih, N. and Warokka, A., 2019. Acceptance and penetration of Bitcoin: the role of psychological distance and national culture. SAGE Open. doi.org/10.1177/2158244019865813. 
Ali, M., Nelson J., Shea, R. and Freedman, M.J. 2016. Blockstack: A Global Naming and Storage System Secured by Blockchains. In: Proceedings of the 2016 USENIX Annual Technical Conference. USENIX ATC Conference 2016. Denver, USA, June 22-24, 2016. Berkeley: USENIX Association. pp.181-194.

Alshamsi, A. and Andras, P., 2019. User perception of Bitcoin usability and security across novice users. International Journal of Human-Computer Studies, 126, pp.94-110.

Blockchain.info, 2019. Bitcoins in circulation. [online] Available at: <https://blockchain.info> [Accessed Sept. 19, 2019].

Chen, D., 2018. Utility of the Blockchain for Climate Mitigation. Journal of The British Blockchain Association, 1(1), pp.75-80. doi10.31585/jbba-1-1-(6)2018.

de Vries, A., 2018. Bitcoin's Growing Energy Problem. Joule, 2, pp.801-809. [online] Available at: <https://www.cell.com/action/showPdf?pii=S2542-4351\%2818\% 2930177-6> [Accessed 4 April 2019].

de Vries, A., 2019a. Bitcoin Energy Consumption Index. [online] Available at: $<$ https://digiconomist.net/bitcoin-energy-consumption> [Accessed 6 September 2019].

de Vries, A., 2019b. Bitcoin Electronic Waste Monitor. [online] Available at: <https://digiconomist.net/bitcoin-electronic-waste-monitor/> [Accessed 6 September 2019.

El Bahrawy, A., Alessandretti, L., Kandler, A., Pastor-Satorrasc, R. and Baronchelli, A., 2017. Evolutionary dynamics of the cryptocurrency market. Royal Society Open Science, 4(11), 170623. doi.org/10.1098/rsos.170623.

Fan, J., Yi, L.T. and Shu, J.W., 2013. Research on the technologies of Byzantine system. Journal of Software, 24(6), pp.1346-1360.

Fedotova, N. and Veltri, L., 2006. Byzantine Generals problem in the light of P2P computing. In: Proceedings of the Third IEEE International Conference on Mobile \& Ubiquitous Systems: Networking \& Services. MOBIQUITOUS 2006, San Jose, CA, 17 July 2006 - 21 July 2006. Washington: IEEE Computer Society. pp.1-5.

Giungato, P., Rana, R.L., Tarabella, A. and Tricase, C., 2017. Current Trends in Sustainability of Bitcoins and Related Blockchain Technology. Sustainability, 9(12), 2214. doi.org/10.3390/su9122214.

Green, J. and Newman, P., 2017. Citizen utilities: The emerging power paradigm. Energy Policy, 105, pp.283-293.

International Energy Agency (IEA), 2017. Digitalization \& Energy. [online] Available at: <https://www.iea.org/publications/freepublications/publication/DigitalizationandEner gy3.pdf> [Accessed 3 April 2019].

Karger, D., Lehman, E., Leighton, T., Levine, M., Lewin, M. and Panigrahy, R., 1997. Consistent Hashing and Random Trees: Distributed Caching Protocols for Relieving Hot Spots on the World Wide Web. In: Proceedings of the Twenty-Ninth ACM Symposium on Theory of Computing. STOC '97 Conference, El Paso, Texas, May 04 06, 1997. New York: ACM Press. pp.654-663.

Lamport, L., 1983. The Weak Byzantine Generals Problem. Journal of the Association for Computing Machinery, 30(3), pp.668-676.

Michel, A. and Hudon, M., 2015. Community currencies and sustainable development: A systematic review. Ecological Economics, 116, pp.160-171. 
Mikroyannidis, A., Domingue, J., Bachler, M. and Quick, K., 2018. A Learner-Centred Approach for Lifelong Learning Powered by the Blockchain. In: T. Bastiaens (ed.). edMedia+Innovate Learning. World Conference on Educational Media and Technology. Amsterdam: EdMedia. pp.1388-1393.

Rana, R.L., Giungato, P., Tarabella, A. and Tricase, C., 2019. Sustainability of bitcoins and blockchain. In: Proceedings of BASIQ International Conference on New Trends in Sustainable Business and Consumption. BASIQ International Conference, 30 May -1 June 2019, Bari, Italy, pp.771-777.

Reischuk, R., 1985. A new solution for the byzantine Generals problem. Information and Control, 64, pp.23-42.

Rutkin, A., 2016. Blockchain-based micro grid gives power to consumers in New York. New Scientist. [online] Available at: <https://www.newscientist.com/article/2079334blockchain-basedmicrogrid- gives-power-to-consumers-in-new-york/?Utm_source= NSNS \& utm_ medium=SOC \& utm_campaign=hoot \& cmpid=SOC\%7CNSNS\% 7C2016-GLOBALhoot> [Accessed 05 April 2019].

Subramanian, H., 2018. Decentralized Blockchain-Based Electronic Marketplaces. Communications of the ACM, 61(1), pp.78-84.

Sun, J., Yan, J., Zhang, K.Z.K., 2016. Blockchain-based sharing services: What blockchain technology can contribute to smart cities. Financial Innovation, 2(26), pp.1-9.

Tapscott, D. and Tapscott, A., 2017. How blockchain will change organizations. MIT Sloan Management Review, 58(2), pp.10-13.

Vranken, H., 2017. Sustainability of bitcoin and blockchains. Current opinion in environmental sustainability, 28, pp.1-9.

Xia, Q., Sifah, E.B., Smahi, A., Amofa, S. and Zhang, X., 2017. BBDS: Blockchain-based data sharing for electronic medical records in cloud environments. Information Switzerland, 8(2), pp.44-45.

Wang, N., Zhou, X., Lu X., Guan, Z. Wu, L., Du, X. and Guizani, M., 2019. When Energy Trading Meets Blockchain in Electrical Power System: The State of the Art. Apply Science, 9(1561), pp.1-31. 